\title{
Shih Tzu
}

National Cancer Institute

\section{Source}

National Cancer Institute. Shih Tzu. NCI Thesaurus. Code C53943.

The Shih Tzu is a small, sturdy dog with a double coat of long hair lined with a woolly undercoat. It has large, round eyes and pendant ears. The head is rounded, with a profuse beard and mustache, short hairy muzzle, and black nose (except in liver-colored dogs which have liver noses). Height: Up to 11 inches $(28 \mathrm{~cm}$.) Weight: 9-16 pounds (4-7 kg.) 